\title{
A COMBINATORIAL CORRESPONDENCE RELATED TO GÖLLNITZ' (BIG) PARTITION THEOREM AND APPLICATIONS
}

\author{
KRISHNASWAMI ALLADI
}

\begin{abstract}
In recent work, Alladi, Andrews and Gordon discovered a key identity which captures several fundamental theorems in partition theory. In this paper we construct a combinatorial bijection which explains this key identity. This immediately leads to a better understanding of a deep theorem of Göllnitz, as well as Jacobi's triple product identity and Schur's partition theorem.
\end{abstract}

\section{INTRODUCTION}

One of the deepest results in the theory of partitions is a theorem of Göllnitz [9] proved in 1967:

Theorem G. Let $B(n)$ denote the number of partitions of $n$ into distinct parts $\equiv 2,4$ or $5(\bmod 6)$.

Let $C(n)$ denote the number of partitions of $n$ in the form $m_{1}+m_{2}+\cdots+m_{\nu}$, no part equal to 1 or 3 , and such that $m_{i}-m_{i+1} \geq 6$ with strict inequality if $m_{i} \equiv 0$, 1 or $3(\bmod 6)$.

Then $B(n)=C(n)$.

The purpose of this paper is to provide a combinatorial correspondence (bijection) which will explain the key identity (1.2) below from which Theorem $\mathrm{G}$ and extensions of it obtained by Göllnitz ([9], Satze 4.8 and 4.10) fall out as special cases. It had remained an open problem for nearly thirty years to give a combinatorial approach to Göllnitz' theorem. In this paper we first obtain a reformulation of Theorem G (see Theorem 2 of $\$ 3$ ) and then construct a bijection to explain this reformulation. The bijection is set up in $\S 5$ as a consequence of a combinatorial study of an identity of Sylvester [11] in $\S 4$. This approach has several applications, including a new interpretation for the Jacobi Triple Product Identity (see §6).

Göllnitz' proof [9] of the equality $B(n)=C(n)$ was extremely complicated and the details are forbidding; but he succeeded in obtaining the refinement

$$
B(n ; \nu)=C(n ; \nu) .
$$

In $(1.1), B(n ; \nu)$ and $C(n ; \nu)$ denote the number of partitions counted by $B(n)$ and $C(n)$ respectively with the additional restriction that there are precisely $\nu$ parts and the convention that parts $\equiv 0,1$ and $3(\bmod 6)$ are counted twice.

Received by the editors September 1, 1995.

1991 Mathematics Subject Classification. Primary 05A17, 05A19; Secondary 11P83.

Key words and phrases. Partitions, Göllnitz' theorem, distinct parts, weighted words, Sylvester's identity, sliding operation.

Research supported in part by National Science Foundation grant DMS 9400191.

(C)1997 American Mathematical Society 
Andrews gave two proofs of Theorem G (and (1.1)), both of them simpler than Göllnitz' original proof. Andrews' first proof [5] was by the method of generating functions; his second proof (see [7], §10) made use of computer algebra to simplify certain calculations. In [7] Andrews asked for a proof which would lend more insight into the equality (1.1).

In a recent paper [3], Andrews, Gordon and I obtained a substantial generalization and refinement of Theorem $\mathrm{G}$ involving three free parameters $a, b$ and $c$, by means of a new technique called the method of weighted words. This method and the principal results of [3] are described in $\S 2$. In [3] we viewed Theorem G as emerging out of the key identity

$$
\sum_{i, j, k} a^{i} b^{j} c^{k} \sum_{\substack{i=\alpha+\delta+\epsilon \\ j=\beta+\delta+\phi \\ k=\gamma+\epsilon+\phi}} \frac{q^{T_{s}+T_{\delta}+T_{\epsilon}+T_{\phi-1}}\left(1-q^{\alpha}\left(1-q^{\phi}\right)\right)}{(q)_{\alpha}(q)_{\beta}(q)_{\gamma}(q)_{\delta}(q)_{\epsilon}(q)_{\phi}}=(-a q)_{\infty}(-b q)_{\infty}(-c q)_{\infty}
$$

under the transformations

$$
\begin{aligned}
\text { (dilation) } q & \mapsto q^{6}, \\
\text { (translations) } a & \mapsto q^{-4}, b \mapsto b q^{-2}, c \mapsto c q^{-1} .
\end{aligned}
$$

In (1.2) and elsewhere, $s=\alpha+\beta+\gamma+\delta+\epsilon+\phi$ and $T_{m}=m(m+1) / 2$. Also in (1.2) we have made use of standard notation

$$
(a)_{n}=(a ; q)_{n}=\prod_{j=0}^{n-1}\left(1-a q^{j}\right),
$$

for a complex number $a$ and a positive integer $n$, and

$$
(a)_{\infty}=\lim _{n \rightarrow \infty}(a)_{n}=\prod_{j=0}^{\infty}\left(1-a q^{j}\right), \quad \text { for } \quad|q|<1 .
$$

The depth of (1.2) becomes clear when one sees that its proof requires not only Watson's $q$-analog of Whipple's theorem but also the ${ }_{6} \psi_{6}$ summation of Bailey (see [3]). We believe that this approach does provide insight into Theorem $\mathrm{G}$ and (1.1), and we will exploit it here to provide a combinatorial bijection for (1.2).

Usually partition identities which permit refinements can be proved combinatorially because the refinements naturally yield the required bijection. For example, there are combinatorial proofs available for Schur's famous 1926 partition theorem (see Bressoud [8] or Alladi-Gordon [4]). Although refinements and extensions of Göllnitz' Theorem G were known [9], no bijection was ever found. The principal idea here is that instead of viewing the transformations (1.3) as yielding the most interesting special case of the key identity (1.2), we show that (1.2) is best understood combinatorially by applying the transformations

$$
\begin{aligned}
\text { (dilation) } q & \mapsto q^{3}, \\
\text { (translations) } a & \mapsto a q^{-2}, b \mapsto b q^{-1}, c \mapsto c .
\end{aligned}
$$

This leads to the reformulation of Theorem $\mathrm{G}$ as Theorem 2 in $\S 3$. It is then possible to construct a bijection (see $\S 5$ ) converting the partitions counted by the series in (1.2) to those counted by the product, which in this case is

$$
\left(-a q ; q^{3}\right)_{\infty}\left(-b q^{2} ; q^{3}\right)_{\infty}\left(-c q^{3} ; q^{3}\right)_{\infty}
$$


There are several advantages in this approach. For instance, Schur's theorem falls out from Theorem 2 by setting $c=0$, and Jacobi's Triple Product Identity can be obtained by choosing $c=-1$ and $a b=1$ (see $\S 6$ ).

We conclude this section by mentioning some notation and conventions. For a partition $\pi: b_{1}+b_{2}+\cdots+b_{\nu}$ with $b_{1} \geq b_{2} \geq \cdots \geq b_{\nu}$, we let $\sigma(\pi)$ denote the sum of the parts of $\pi$ and $\nu(\pi)=\nu$, the number of parts of $\pi$. By using subscripts we indicate counting the number of parts of a certain type; for example, $\nu_{a}(\pi)$ will denote the number of $a$-parts of $\pi$. Also, when we say $\pi$ is a partition with minimal difference $k$, we mean that the parts of $\pi$ differ by at least $k$.

\section{THE METHOD OF WEIGHTED WORDS}

Here we summarize the principal ideas and results in [3].

We consider integers $n \geq 2$ as occurring in six colors, three of which, $a, b$ and $c$, are primary colors, and three colors $a b, a c$ and $b c$ are secondary. The integer 1 occurs only in the primary colors $a, b$ and $c$. The letters $a, b, c, d, e$ and $f$ play a dual role. On the one hand they represent the colors of the integers; on the other, they are free parameters.

An integer occuring in a particular color is denoted by a symbol whose subscript is the integer and whose letter represents the color. For example, the integer 7 in color $a$ is represented by $a_{7}$ and $d_{6}=a b_{6}$ is the integer 6 in color $d=a b$. The subscript of a symbol is its weight. The gap between symbols is the absolute value of the difference between their weights. Thus the gap between $a_{6}$ and $d_{9}$ is 3 .

A word is a finite ordered collection of symbols. In order to discuss partitions, we need to put an ordering on the symbols, because a partition is a word in which the symbols are in decreasing order according to this ordering. The symbols occurring in a partition are called parts; thus if $a_{j}$ and $b_{k}$ occur in a partition $\pi$, we call $a_{j}$ an $a$-part, $b_{k}$ a $b$-part, and so on.

The ordering we first choose is

$$
\text { Scheme 1: } a_{1}<b_{1}<c_{1}<d_{2}<e_{2}<a_{2}<f_{2}<b_{2}<c_{2}<d_{3}<e_{3}<\ldots
$$

The reason for this is that under the transformations (1.3), the symbols become

$$
\begin{aligned}
& a_{j}=6 j-4, b_{j}=6 j-2, c_{j}=6 j-1, \\
& d_{j}=6 j-6, e_{j}=6 j-5, f_{j}=6 j-3,
\end{aligned}
$$

and so the ordering in (2.1) becomes

$$
2<4<5<6<7<8<9<10<11<12<\ldots,
$$

the standard ordering among the integers. Note that the integers 1 and 3 are absent from the list (2.3); this will explain the absence of 1 and 3 as parts among the partitions counted by $C(n)$ in Theorem $\mathrm{G}$.

Other orderings are possible, and indeed there are $6 !=720$ of them, corresponding to the various permutations of the six colors $a, b, c, d, e$ and $f$ ! In [3] there is a discussion of these other orderings and the companion results to Theorem $G$ that are generated by these orderings. For our purpose, one other ordering, namely, Scheme 2 given below in (2.8), will be relevant.

We may represent a partition $\pi$ as a collection of symbols in decreasing order or as a sum. Thus $\pi=c_{3} b_{3} d_{3} a_{2} a_{2} e_{2} b_{1} a_{1}$ is a partition which could also be written as $\pi=c_{3}+b_{3}+d_{3}+a_{2}+a_{2}+e_{2}+b_{1}+a_{1}$. For a partition $\pi, \sigma(\pi)$ is the sum of the weights of $\pi$, and we think of $n=\sigma(\pi)$ as being partitioned in terms of the colored 
integers. So, in this example, $n=\sigma(\pi)=17$. By $\nu_{a}(\pi)$ we mean the number of $a$-parts of $\pi$, by $\nu_{b}(\pi)$ the number of $b$-parts of $\pi$, and so on. In this example, $\nu_{a}(\pi)=3, \nu_{b}(\pi)=2, \nu_{c}(\pi)=1, \nu_{d}(\pi)=1, \nu_{e}(\pi)=1, \nu_{f}(\pi)=0$ and $\nu(\pi)=8$.

For symbols with the same weight, observe that the colors in (2.1) occur in the following order:

$$
\text { Scheme 1: } d<e<a<f<b<c \text {. }
$$

Given two colors, we use (2.4) to determine which is of lower order. This notion is of importance for the partitions defined below.

Now consider partitions $\pi=m_{1}+m_{2}+\ldots$ using symbols from (2.1) such that the gap between the parts $m_{\ell}$ is $\geq 1$ with the added restriction that the gap between consecutive parts $m_{\ell}$ and $m_{\ell+1}$ is $\geq 2$ if

$$
\begin{aligned}
& m_{\ell} \text { is lower order and } m_{\ell+1} \text { is of higher order, } \\
& \text { or if } m_{\ell} \text { and } m_{\ell+1} \text { are of the same secondary color. }
\end{aligned}
$$

We call such partitions $\pi$ Type 1 partitions. Under the transformations (1.3), Type 1 partitions become the partitions of the type counted by $C(n)$ in Theorem $\mathrm{G}$. The principal result proved in $[3]$ is

Theorem 1. Let $B(n ; i, j, k)$ be the number of vector partitions $\pi^{\prime}=\left(\pi_{1} ; \pi_{2} ; \pi_{3}\right)$ of $n$ such that $\pi_{1}, \pi_{2}$ and $\pi_{3}$ have distinct a-parts, b-parts and c-parts respectively and also $\nu\left(\pi_{1}\right)=i, \nu\left(\pi_{2}\right)=j$ and $\nu\left(\pi_{3}\right)=k$.

Let $C(n ; \alpha, \beta, \gamma, \delta, \epsilon, \phi)$ be the number of Type 1 partitions $\pi$ of $n$ such that $\nu_{a}(\pi)=\alpha, \nu_{b}(\pi)=\beta, \ldots, \nu_{f}(\pi)=\phi$. Then

$$
B(n ; i, j, k)=\sum_{\substack{i=\alpha+\delta+\epsilon \\ j=\beta+\delta+\phi \\ k=\gamma+\epsilon+\phi}} C(n ; \alpha, \beta, \gamma, \delta, \epsilon, \phi) .
$$

Theorem 1 is proved in [3] by showing that the generating functions of $B$ and $C$ are equal. The generating function of $B(n ; i, j, k)$ is clear. It is

$$
\sum_{n=0}^{\infty} B(n ; i, j, k) a^{i} b^{j} c^{k} q^{n}=(-a q)_{\infty}(-b q)_{\infty}(-c q)_{\infty} .
$$

The generating function of $C(n ; \alpha, \beta, \gamma, \delta, \epsilon, \phi)$ is much more difficult to determine. We show in [3] that for a given $\alpha, \beta, \gamma, \delta, \epsilon$ and $\phi$,

$$
\sum_{n=0}^{\infty} C(n ; \alpha, \beta, \gamma, \delta, \epsilon, \phi) q^{n}=\frac{q^{T_{s}+T_{\delta}+T_{\epsilon}+T_{\phi-1}}\left(1-q^{\alpha}\left(1-q^{\phi}\right)\right)}{(q)_{\alpha}(q)_{\beta}(q)_{\gamma}(q)_{\delta}(q)_{\epsilon}(q)_{\phi}} .
$$

From (2.6) and (2.7) we see that Theorem 1 is equivalent to (1.2). Under the transformations (1.3), Theorem 1 yields a multiparameter refinement of Theorem G.

The combinatorial interpretation for the left hand side of (1.2) that has been given above is that it is the generating function for Type 1 partitions given by (2.5) with the ordering provided by (2.1). Other combinatorial interpretations are possible by considering different orderings. For example, consider the ordering given by

$$
\text { Scheme 2: } a_{1}<b_{1}<d_{2}<c_{1}<e_{2}<a_{2}<f_{2}<b_{2}<d_{3}<c_{2}<\ldots
$$


Under the transformations (2.2), the ordering (2.8) becomes

$$
2<4<6<5<7<8<9<10<12<11<\ldots
$$

which is not the natural ordering among the integers. In order to define Type 2 partitions using Scheme 2, it will be more convenient to reformulate the definition of Type 1 partitions. For this purpose consider the full list of symbols in Scheme 1 given by

$$
\text { Scheme } 1: \underline{e}_{1}<a_{1}<\underline{f}_{1}<b_{1}<c_{1}<d_{2}<e_{2}<a_{2}<f_{2}<b_{2}<c_{2}<\ldots
$$

Note that in (2.10) the symbol $d_{1}$ is omitted because its value is 0 under the transformations (2.2). Also the symbols $e_{1}$ and $f_{1}$ are underlined in (2.10) to indicate that they are absent in (2.1) and therefore will never appear as parts. We refer to the transformations (2.2) as standard transformations.

Now let $x_{m}=x_{m}^{(1)}$ denote the symbol occupying position $m$ in (2.10). That is, $x_{1}=\underline{e}_{1}, x_{2}=a_{2}, x_{3}=\underline{f}_{1}$, and so on. With this notation, Type 1 partitions are those of the form $x_{m_{1}}+x_{m_{2}}+\ldots$ such that

$$
m_{\ell}-m_{\ell+1} \geq 6 \text { with strict inequality if } x_{m_{\ell}} \text { is of secondary color. }
$$

We refer to the inequalities in (2.11) as standard gap conditions.

Now consider the full list of symbols in Scheme 2 given by

Scheme 2: $\underline{e}_{1}<a_{1}<\underline{f}_{1}<b_{1}<d_{2}<c_{1}<e_{2}<a_{2}<f_{2}<b_{2}<d_{3}<c_{2}<\ldots$

Let $x_{m}=x_{m}^{(2)}$ denote the symbol occupying position $m$ in (2.12). Then Type 2 partitions are those of the form $x_{m_{1}}+x_{m_{2}}+\ldots$ such that $x_{m_{\ell}}=x_{m_{\ell}}^{(2)}$ satisfy the standard gap conditions in (2.11).

Next let $C_{2}(n ; \alpha, \beta, \gamma, \delta, \epsilon, \phi)$ denote the number of Type 2 partitions $\pi$ of $n$ such that $\nu_{a}(\pi)=\alpha, \nu_{b}(\pi)=\beta, \ldots, \nu_{f}(\pi)=\phi$. In [3] it was shown combinatorially that

$$
C(n ; \alpha, \beta, \delta, \gamma, \epsilon, \phi)=C_{2}(n ; \alpha, \beta, \gamma, \delta, \epsilon, \phi) .
$$

Thus the series in (1.2) may also be interpreted as the generating function of $C_{2}(n ; \alpha, \beta, \gamma, \delta, \epsilon, \phi)$. Under the transformations (2.2), Scheme 2 and (2.13) yield the following companion result to Theorem $\mathrm{G}$, as was noticed in [3]:

Theorem $\mathbf{G}_{2}$. Let $C_{2}(n)$ denote the number of partitions of $n$ of the form $m_{1}+$ $m_{2}+\ldots$ such that no $m_{\ell}=1$ or 3 and $m_{\ell}-m_{\ell+1} \geq 7,6,7,6,5$ or 8 if $m_{\ell} \equiv$ $1,2,3,4,5$ or $0(\bmod 6)$. Let $C(n)$ be as in Theorem $\mathrm{G}$. Then

$$
C(n)=C_{2}(n) .
$$

Note that the two partition functions in (2.13) correspond to the left side of (1.2) and so they are combinatorially equivalent in an elementary way (see [3]). What is difficult is to produce a combinatorial correspondence which would explain the equality between the series and the product in (1.2). This is achieved in the next three sections by considering the effect of the transformations (1.4) on (1.2). 


\section{A special Dilation}

In terms of the symbols introduced in $\S 2$ in connection with the method of weighted words, the effect of the transformations (1.4) is to convert them to

$$
\begin{gathered}
a_{n} \mapsto 3 n-2, b_{n} \mapsto 3 n-1, c_{n} \mapsto 3 n, \\
d_{n}=a b_{n} \mapsto 3 n-3, e_{n}=a c_{n} \mapsto 3 n-2, f_{n}=b c_{n} \mapsto 3 n-1 .
\end{gathered}
$$

Thus, under (3.1), Scheme 1 ordering in (2.1) becomes

$$
1_{a}<2_{b}<3_{c}<3_{a b}<4_{a c}<4_{a}<5_{b c}<5_{b}<6_{c}<6_{a b}<\ldots,
$$

where in (3.2) we have reversed the convention by making the subscript represent the color of the integer. The important thing to note is that in (3.2) each integer $n \geq 3$ occurs in two colors, one primary and one secondary, the primary color given by the residue class $(\bmod 3)$ as in (1.5). The convenient feature of the ordering (3.2) is that if $m$ and $n$ are (uncolored) positive integers with $m<n$, then $m<n$ in (3.2) regardless of the color of $m$ and $n$. It is to be noted that in (3.2), for integers $n \not \equiv 0(\bmod 3)$ with $n>3, n$ having secondary color is less than $n$ having primary color. On the other hand, if $n \equiv 0(\bmod 3)$, then $n$ with primary color is less than $n$ with secondary color. This twist creates a slight complication in the definition of weights in (3.7)-(3.9) below.

Actually, under the transformations (1.4), Scheme 2 behaves better. Indeed under (3.1), the ordering in (2.8) becomes

$$
1_{a}<2_{b}<3_{a b}<3_{c}<4_{a c}<4_{a}<5_{b c}<5_{b}<6_{a b}<6_{c}<\ldots
$$

The added convenience of (3.3) over (3.2) is that in (3.3), for all integers $n \geq 3, n$ with secondary color is less than $n$ with primary color. Therefore we first discuss the partition theorem that comes out of Theorem 1 and (2.14) under the effect of (1.4) and the Scheme 2 ordering in (3.3).

Let $\tilde{\pi}$ be a partition with (uncolored) parts $m_{1}+m_{2}+\cdots+m_{\nu}$ such that the difference between parts is $\geq 3$. Each part $m_{i}$ of $\tilde{\pi}$ will be counted with a weight $w_{2}\left(m_{i} ; \tilde{\pi}\right)=w_{2}\left(m_{i}\right)$ which will be determined by the residue class $(\bmod 3)$ of $m_{i}$ and the difference $m_{i}-m_{i+1}$ as given by the table below. We adopt the convention that $m_{\nu+1}=-1$ to determine the weight $w_{2}\left(m_{\nu}\right)$.

$\begin{array}{ccc}\text { congruence } & \text { gap } & \text { weight } w_{2}\left(m_{i}\right) \\ m_{i} \equiv 1(\bmod 3) & m_{i}-m_{i+1} \leq 3 & a \\ m_{i} \equiv 2(\bmod 3) & m_{i}-m_{i+1} \leq 3 & b \\ m_{i} \equiv 0(\bmod 3) & m_{i}-m_{i+1} \leq 3 & c \\ m_{i} \equiv 1(\bmod 3) & m_{i}-m_{i+1}>3 & a+a c \\ m_{i} \equiv 2(\bmod 3) & m_{i}-m_{i+1}>3 & b+b c \\ m_{i} \equiv 0(\bmod 3) & m_{i}-m_{i+1}>3 & c+a b\end{array}$

The weight $w_{2}(\tilde{\pi})$ of the partition $\tilde{\pi}$ is defined multiplicatively as the product of the weights of its parts. Thus for the partition

$$
\tilde{\pi}: 18+15+11+8+3
$$

the weights of its parts are

$$
w_{2}(18)=c, w_{2}(15)=c+a b, w_{2}(11)=b, w_{2}(8)=b+b c \text { and } w_{2}(3)=c+a b .
$$


Thus, in the above example,

$$
w_{2}(\tilde{\pi})=c(c+a b) b(b+b c)(c+a b) .
$$

So the equality $B(n)=C_{2}(n)$ provided by Theorem 1 and (2.13) becomes

Theorem 2. Let $\mathcal{D}(n)$ denote the set of partitions of $n$ into distinct parts. For $\pi^{\prime} \in \mathcal{D}(n)$, let $\nu_{i}\left(\pi^{\prime}\right)$ denote the number of parts $\pi^{\prime}$ that are $\equiv i(\bmod 3)$, for $i=$ $1,2,3$.

Let $\mathcal{D}_{3}(n)$ denote the set of partitions of $n$ with difference $\geq 3$ between parts. Then

$$
\sum_{\pi^{\prime} \in \mathcal{D}(n)} a^{\nu_{1}\left(\pi^{\prime}\right)} b^{\nu_{2}\left(\pi^{\prime}\right)} c^{\nu_{3}\left(\pi^{\prime}\right)}=\sum_{\tilde{\pi} \in \mathcal{D}_{3}(n)} w_{2}(\tilde{\pi}) .
$$

The table below illustrates the equality in Theorem 3 for the integer $n=9$. It is easily checked that the sum of weights in the second and fourth columns of the table are the same.

\begin{tabular}{|c|c|c|c|}
\hline$\pi^{\prime} \in \mathcal{D}(9)$ & $a^{a_{1}\left(\pi^{\prime}\right)} b^{\nu_{2}\left(\pi^{\prime}\right)} c^{\nu_{3}\left(\pi^{\prime}\right)}$ & $\tilde{\pi} \in \mathcal{D}_{3}(9)$ & $w_{2}(\tilde{\pi})$ \\
\hline 9 & $c$ & 9 & $\overline{c+a b}$ \\
\hline $8+1$ & $a b$ & $8+1$ & $(b+b c) a$ \\
\hline $7+2$ & $a b$ & $7+2$ & $(a+a c) b$ \\
\hline $6+3$ & $c^{2}$ & $6+3$ & $c(c+a b)$ \\
\hline $6+2+1$ & $a b c$ & & \\
\hline $5+4$ & $a b$ & & \\
\hline $5+3+1$ & $a b c$ & & \\
\hline $4+3+2$ & $a b c$ & & \\
\hline
\end{tabular}

\section{TABLE 1}

The equality $B(n)=C(n)$ in Theorem 1 is given by Scheme 1 ordering (2.1). For the corresponding equality given by (3.1) and the Scheme 1 ordering in (3.2), the definition of weights $w_{1}(\tilde{\pi})$ for partitions $\tilde{\pi}=m_{1}+m_{2}+\ldots m_{\nu}$ with minimal difference 3 is a bit more complicated as given below:

$$
\begin{aligned}
& \text { If } m_{i}-m_{i+1}=3 \text { and }\left\{\begin{array}{l}
m_{i} \equiv 1(\bmod 3), \text { then } w_{1}\left(m_{i}\right)=a ; \\
m_{i} \equiv 2(\bmod 3), \text { then } w_{1}\left(m_{i}\right)=b ; \\
m_{i} \equiv 0(\bmod 3), \text { then } w_{1}\left(m_{i+1}\right)=c .
\end{array}\right. \\
& \text { If } m_{i}-m_{i+1}=3, m_{i} \equiv m_{i+1} \equiv 3(\bmod 3) \text { and } m_{i-1}-m_{i}>3,
\end{aligned}
$$

The weight $w_{1}(\tilde{\pi})$ of the partition $\tilde{\pi}$ is defined multiplicatively to be the product of the weights of its parts. Here also we adopt the convention $m_{\nu+1}=-1$ to determine $w_{1}\left(m_{\nu}\right)$. In addition we set $m_{0}=\infty$ to determine $w_{1}\left(m_{1}\right)$.

The main difference between $w_{1}(\tilde{\pi})$ and $w_{2}(\tilde{\pi})$ is that in $(3.7)$, when $m_{i} \equiv$ $3(\bmod 3)$, it is $w_{1}\left(m_{i+1}\right)=c$. This is the reason why $(3.8)$ is necessary. This slight 
complication in the definition of weights $w_{1}\left(m_{i}\right)$ is caused by the quirk that in (3.2), only for integers $n \equiv 0(\bmod 3), n$ with primary color is less than $n$ with secondary color. For a partition $\tilde{\pi}$ with minimal difference 3 , although the weights of its parts $w_{1}\left(m_{i}\right)$ and $w_{2}\left(m_{i}\right)$ could be different, it is not difficult to see that $w_{1}(\tilde{\pi})=w_{2}(\tilde{\pi})$. For example, with $\tilde{\pi}$ as in (3.4) we have

$$
w_{1}(18)=c+a b, w_{1}(15)=c, w_{1}(11)=b, w_{1}(8)=b+b c, \text { and } w_{1}(3)=c+a b,
$$

which is different from (3.5). But then

$$
w_{1}(\tilde{\pi})=(c+a b) c \cdot b \cdot(b+b c) \cdot(c+a b)=w_{2}(\tilde{\pi}),
$$

with $w_{2}(\tilde{\pi})$ given by (3.6). So we have

Theorem 3. Let $\tilde{\pi}$ be a partition with minimal difference 3 . Then

$$
w_{1}(\tilde{\pi})=w_{2}(\tilde{\pi}) .
$$

Consequently, in Theorem 2, $w_{2}(\tilde{\pi})$ could be replaced by $w_{1}(\tilde{\pi})$.

In the next two sections we will produce a combinatorial bijection involving partitions into distinct parts which will explain Theorem 2.

\section{Sylvester's IDENTITY}

By analyzing partitions into distinct parts in terms of Durfee squares, Sylvester [11] showed in his classic paper of 1882 that

$$
(-z q)_{\infty}=1+\sum_{n=1}^{\infty} \frac{z^{n} q^{\left(3 n^{2}-n\right) / 2}\left(1+z q^{2 n}\right)(-z q)_{n-1}}{(q)_{n}} .
$$

In [1] we obtained a new combinatorial interpretation for (4.1) (see Theorems 4 and 5 below), and this has paved the way for the bijection in $\S 5$ explaining Theorem 2 .

Consider a partition $\tilde{\pi}$ into $\nu$ parts $m_{1}>m_{2}>\ldots m_{\nu}$ with minimal difference 3. Put $m_{\nu+1}=-1$. Suppose the partition $\tilde{\pi}$ has exactly $k$ gaps $m_{i}-m_{i+1} \geq 4$ for $1 \leq i \leq \nu$. Then define the weight $w(\tilde{\pi})$ of $\tilde{\pi}$ to be

$$
w(\tilde{\pi})=z^{\nu}(1+z)^{k} .
$$

In [1] it was shown that the right hand side of (4.1) is the generating function for partitions $\tilde{\pi}$ with minimal difference 3 between parts such that each $\tilde{\pi}$ is counted with weight $w(\tilde{\pi})$ as in (4.2). Thus (4.1) has the following partition interpretation.

Theorem 4. Let $Q(n ; k)$ denote the number of partitions $\pi$ of $n$ into distinct parts with $\nu(\pi)=k$.

Let $g_{3}(n ; \nu, k)$ denote the number of partitions $\tilde{\pi}$ of $n$ into parts with minimal difference 3 such that $\nu(\tilde{\pi})=\nu$ and $\tilde{\pi}$ has exactly $k$ gaps $\geq 4$ as described above.

Then

$$
\sum_{k} z^{k} Q(n ; k)=\sum_{\nu} \sum_{k} z^{\nu}(1+z)^{k} g_{3}(n ; \nu, k) .
$$

In Theorem 4 , take $z=1$ and set

$$
g_{3}(n ; k)=\sum_{\nu} g_{3}(n ; \nu, k)
$$


and

$$
Q(n)=\sum_{k} Q(n ; k)
$$

This gives

Theorem 5. $Q(n)=\sum_{k} g_{3}(n ; k) 2^{k}$.

The proof of Theorem 5 in [1] utilized the concept of imbeddings to convert vector partitions into ordinary partitions. These imbeddings are usually not oneto-one mappings. Although every vector bi-partition under an imbedding gives rise to a unique partition, several vector partitions could give rise to the same partition. Identity (4.1) first provides a natural correspondence between partitions $\pi$ into distinct parts and certain bi-partitions $\left(\pi_{1} ; \pi_{2}\right)$. It turns out that, under imbeddings, there are $2^{k}$ bi-partitions which yield a partition $\tilde{\pi}$ with minimal difference 3 between parts and having $k$ gaps $\geq 4$. Thus Theorem 5 is really a consequence of the following result proved in [1].

Theorem 6. Let $\tilde{\pi}$ be a partition counted by $g_{3}(n ; k)$. Then there are $2^{k}$ partitions $\pi$ counted by $Q(n)$ which naturally correspond to $\tilde{\pi}$.

Theorem 2 is a three-parameter refinement of Theorems 4 and 5. Setting $a=$ $b=c=z$ in the weight formula for $w_{2}(\tilde{\pi})$ given by the table in $\S 3$ yields the weight formula (4.2). The bijection for Theorem 2 established in the next section is different from the construction involving imbeddings for Theorem 4 given in [1], but it makes use of an idea called the sliding operation which was introduced in [1].

\section{THE BIJECTION}

We begin by defining the sliding operation.

Each partition $\pi$ can be represented by a Ferrers graph where the number of nodes in each row of the graph correspond to the parts of the partition. We make no distinction between a partition $\pi$ and its Ferrers graph.

Every Ferrers graph $\pi$ contains a largest square of nodes starting from the upper left hand corner, called the Durfee square of the partition and denoted by $D(\pi)$. The Ferrers graph and the Durfee square of the partition $\pi: 8+8+7+4+4+2+1$ of the integer 34 are illustrated below.

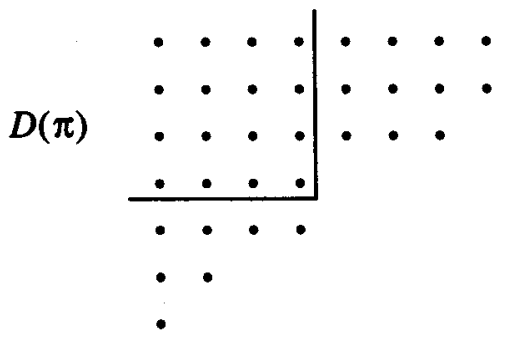

Given a Ferrers graph of a partition $\pi$, we could count nodes along hooks (rectangular bends) starting from the outermost and moving inwards to get a new partition $\tilde{\pi}=\rho(\pi)$ of the same integer. In (5.1) above, the hooks yield the partition $\tilde{\pi}: 14+11+7+2$ of 34 . It is to be noted that the number of parts of $\tilde{\pi}$ equals 
the dimension of the Durfee square, which is the number of nodes on a side of the Durfee square. That is, $\nu(\tilde{\pi})=\operatorname{dim}(D(\pi))$.

By a sliding operation $\psi$ on a partition $\pi$, we mean the removal of certain columns to the right of the Durfee square and the placement of these columns as rows below the Durfee square to form a new Ferrers graph $\pi^{\prime}=\psi(\pi)$. Several sliding operations could be performed on a given $\pi$ to yield many $\pi^{\prime}$. The following are invariants under the sliding operation.

(i) The number of nodes in $\pi$ and $\pi^{\prime}=\psi(\pi)$ are the same.

(ii) The partitions $\pi$ and $\pi^{\prime}=\psi(\pi)$ have the same Durfee square.

(iii) Most importantly, the partition $\rho(\pi)$ obtained by counting nodes in hooks of $\pi$ is the same as the partition $\rho\left(\pi^{\prime}\right)$ obtained from hooks of $\pi^{\prime}$. That is,

$$
\rho(\pi)=\rho(\psi(\pi))
$$

Now consider the Ferrers graph of a partition $\pi$ into distinct parts. This graph $\pi$ contains a Durfee square of $D(\pi)$ of dimension, say, $\nu$. Among the nodes to the right of $D(\pi)$ we can locate an isosceles triangle of $T_{\nu-1}=\frac{\nu(\nu-1)}{2}$ nodes with one of two equal sides of this isosceles triangle being a column of nodes next to the Durfee square. The Durfee square and the triangle of nodes are illustrated below for the partition $\pi: 10+9+7+5+4+2+1$ of 38 into distinct parts. In this example $\operatorname{dim}(D(\pi))=\nu=4$.

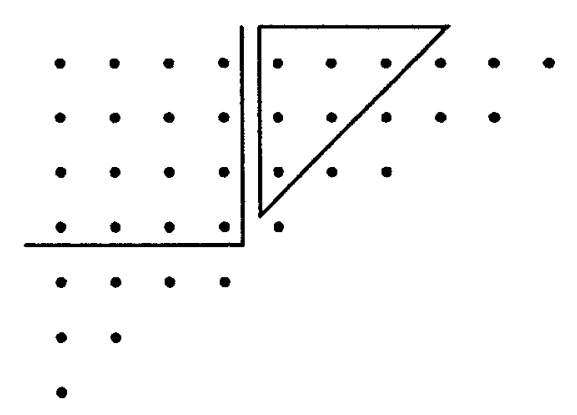

Owing to the presence of the Durfee square and the triangle of nodes in $\pi$, the partition $\tilde{\pi}=\rho(\pi)$ will be a partition with minimal difference 3 . In (5.2), $\tilde{\pi}: 16+12+7+3$ is the resulting partition of 38 with difference $\geq 3$ between parts.

Given a partition $\tilde{\pi}$ into parts with minimal difference 3 , we now determine all Ferrers graphs $\pi^{\prime}$ of partitions into distinct parts for which $\rho\left(\pi^{\prime}\right)=\tilde{\pi}$.

Consider the Ferrers graph $\pi$ of a partition $n_{1}+n_{2}+\cdots+n_{\nu}$ into distinct parts such that $n_{\nu} \geq \nu$, as shown below, having no nodes below the Durfee square. We call such a Ferrers graph (partition) a primary Ferrers graph (partition).

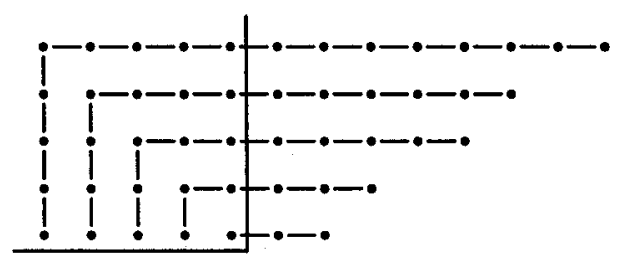


Counting nodes along hooks in such a graph yields a partition $\rho(\pi)=\tilde{\pi}$ with minimal difference 3 between parts. In (5.3) the partition $\pi$ is $13+11+10+8+7$ and $\tilde{\pi}$ is $17+13+10+6+3$.

Conversely, given a partition $\tilde{\pi}: m_{1}+m_{2}+\cdots+m_{\nu}$ with minimal difference 3 between parts, there is a unique primary Ferrers graph $\pi$ of a partition into distinct parts which yields $\tilde{\pi}$. To construct this primary Ferrers graph, first put down a $\nu \times \nu$ square of nodes. Next count nodes along the hooks of this square and attach nodes as necessary to the right of the square to get the parts of $\tilde{\pi}$. Thus we have:

Theorem 7. The number of partitions of $n$ with difference $\geq 3$ between parts is equal to the number of primary partitions of $n$ into distinct parts.

Given $\tilde{\pi}: m_{1}+m_{2}+\cdots+m_{\nu}$ with $m_{i}-m_{i+1} \geq 3$ for $1 \leq i<\nu$, set $m_{\nu+1}=-1$. Construct the primary Ferrers graph $\pi$ as in (5.3) above, which yields $\tilde{\pi}=\rho(\pi)$. Notice that for any $i$ with $1 \leq i \leq \nu$, if $m_{i}-m_{i+1} \geq 4$, then it is possible to remove a column of length $i$ from the portion of the Ferrers graph to the right of the Durfee square and place it below the Durfee square as a row such that the resulting Ferrers graph $\pi^{\prime}$ will represent a partition into distinct parts. Note that if such a column is actually removed and slid down, then $\pi^{\prime}$ will not be a primary Ferrers graph. By circling the appropriate nodes in (5.4) below, we show which columns in (5.3) could actually be moved under the sliding operation.

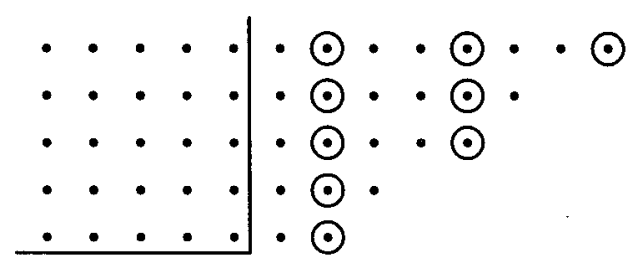

Thus if $m_{i}-m_{i+1} \geq 4$, we may or may not move a column of length $i$ to a position below the Durfee square. This gives us two choices. So, if $\tilde{\pi}: m_{1}+m_{2}+\cdots+m_{\nu}$ has exactly $k$ gaps $m_{i}-m_{i+1} \geq 4$ for $1 \leq i \leq \nu$, then we have $2^{k}$ choices; that is, $2^{k}$ sliding operations can be performed on the primary Ferrers graph $\pi$ for which $\tilde{\pi}=\rho(\pi)$, to yield $2^{k}$ partitions $\pi^{\prime}=\psi(\pi)$ into distinct parts. Because of the invariance (iii), every such partition $\pi^{\prime}$ will yield the same $\tilde{\pi}=\rho\left(\pi^{\prime}\right)$ by counting nodes along hooks. Thus by considering sliding operations on primary Ferrers graphs we have given another proof of Theorem 6 , which in turn implies Theorem 5 . We will now improve this to explain Theorem 2.

Given the Ferrers graph of a partition $\pi^{\prime}$ into distinct parts $n_{1}+n_{2}+\ldots$, assign to each part $n_{i}$ a weight as follows:

$$
\left\{\begin{array}{l}
\text { If } n_{i} \equiv 1(\bmod 3), \text { then } n_{i} \text { has weight } a . \\
\text { If } n_{i} \equiv 2(\bmod 3), \text { then } n_{i} \text { has weight } b . \\
\text { If } n_{i} \equiv 0(\bmod 3), \text { then } n_{i} \text { has weight } c .
\end{array}\right.
$$

So, if $\nu_{j}\left(\pi^{\prime}\right)$ denotes the number of parts of $\pi^{\prime}$ which are $\equiv j(\bmod 3)$, for $j=1,2,3$, then the weight of such a partition defined multiplicatively is

$$
a^{\nu_{1}\left(\pi^{\prime}\right)} b^{\nu_{2}\left(\pi^{\prime}\right)} c^{\nu_{3}\left(\pi^{\prime}\right)},
$$

as on the left side sum in Theorem 2. 
Now we know that all partitions $\pi^{\prime}$ of $n$ into distinct parts are obtained by considering all partitions $\tilde{\pi}$ of $n$ into parts differing by $\geq 3$, constructing the primary Ferrers graphs $\pi$ for which $\tilde{\pi}=\rho(\pi)$, and performing sliding operations on the graphs $\pi$. The parts $m_{1}+m_{2}+\cdots+m_{\nu}$ of $\tilde{\pi}$ arise by counting nodes along hooks of $\pi$. Initially, assign to each part $m_{i}$ of $\tilde{\pi}$ a weight $a, b, c$ as in (5.5), depending on whether $m_{i} \equiv 1,2$ or $3(\bmod 3)$. Suppose for a certain $i, m_{i}-m_{i+1} \geq 4$ with the convention $m_{\nu+1}=-1$. Then it is possible to move a column of length $i$ from the right of $D(\pi)$, the Durfee square of $\pi$, and place it below $D(\pi)$ horizontally to form a graph $\pi^{\prime}$. We know that $\rho\left(\pi^{\prime}\right)=\tilde{\pi}$. Moreover, we note now that the part obtained by counting nodes along the $i^{\text {th }}$ hook of $\pi^{\prime}$ will be $m_{i}$, the $i^{\text {th }}$ part of $\tilde{\pi}$. Since this hook begins from an end point of a row below the Durfee square, we will assign $m_{i}$ the weight

$$
a c, b c \text { or } a b \text { depending on } m_{i} \equiv 1,2 \text { or } 3(\bmod 3),
$$

in addition to the weight previously assigned to $m_{i}$ as in (5.5). Since for parts $m_{i}$ of $\tilde{\pi}$ satisfying $m_{i}-m_{i+1} \geq 4$ we have a choice of moving the column of length $i$, the total weight assigned to $m_{i}$ must be the sum of weights prescribed by (5.5) and (5.6). Thus we have:

$$
\left\{\begin{array}{l}
\text { If } m_{i}-m_{i+1} \geq 4 \text { and } m_{i} \equiv 1(\bmod 3), \text { then weight of } m_{i} \text { is } a+a c . \\
\text { If } m_{i}-m_{i+1} \geq 4 \text { and } m_{i} \equiv 2(\bmod 3), \text { then weight of } m_{i} \text { is } b+b c . \\
\text { If } m_{i}-m_{i+1} \geq 4 \text { and } m_{i} \equiv 0(\bmod 3), \text { then weight of } m_{i} \text { is } c+a b .
\end{array}\right.
$$

If $m_{i}-m_{i+1}<4$, then the weight of $m_{i}$ is as in (5.5). Finally, the weight $w_{2}(\tilde{\pi})$ of $\tilde{\pi}$ is defined multiplicatively to be the product of the weights of its parts, and this is exactly what we see on the right hand side of the equality in Theorem 2 .

This rule of weights can be easily transformed from partitions $\tilde{\pi}$ to partitions $\pi^{\prime}$ into distinct parts as follows. Given a partition $\pi^{\prime}$ into distinct parts, let $\tilde{\pi}=$ $\rho\left(\pi^{\prime}\right)=m_{1}+m_{2}+\ldots$ be the partition with minimal difference 3 obtained from hooks of $\pi^{\prime}$. Suppose the $i^{\text {th }}$ hook of $\pi^{\prime}$, which yields $m_{i}$, begins from an end point below the Durfee square of $\pi^{\prime}$ (equivalently, if $i$ is a part of $\pi^{\prime}$ ), then assign $m_{i}$ a weight $\tilde{w}\left(m_{i}\right)$ as prescribed by (5.6). If the $i^{t h}$ hook of $\pi^{\prime}$ does not begin from an end point below the Durfee square of $\pi^{\prime}$ (equivalently, if $i$ is not a part of $\pi^{\prime}$ ), then assign $m_{i}$ a weight $\tilde{w}\left(m_{i}\right)$ as prescribed by (5.5). Now define the weight $\tilde{w}\left(\pi^{\prime}\right)$ of $\pi^{\prime}$ to be the product of weights assigned to the parts of $\tilde{\pi}$ (not of $\pi^{\prime}$ ). The sum of these weights over all partitions $\pi^{\prime}$ of $n$ into distinct parts is equal to the sum on the right hand side of Theorem 2. Thus Theorem 2 could be reformulated as

Theorem 8. $\sum_{\pi^{\prime} \in \mathcal{D}(n)} a^{\nu_{1}\left(\pi^{\prime}\right)} b^{\nu_{2}\left(\pi^{\prime}\right)} c^{\nu_{3}\left(\pi^{\prime}\right)}=\sum_{\pi^{\prime} \in \mathcal{D}(n)} \tilde{w}\left(\pi^{\prime}\right)$.

The table below gives the set of partitions $\pi^{\prime}$ of 9 into distinct parts, their Ferrers graphs, the partitions $\tilde{\pi}$ they generate, and the weights $w\left(\pi^{\prime}\right)$ and $\tilde{w}\left(\pi^{\prime}\right)$ given by the rules above. The weight (color) of each part is indicated as a subscript.

Notice that the sum of the weights in the second and fifth columns of Table 2 are the same, verifying Theorem 8 for $n=9$.

Next, compare Table 2 with Table 1 in $\S 3$. Observe that the partition $\tilde{\pi}=9$ occurs twice in the fourth column of Table 2. The sum of the weights $\tilde{w}\left(\pi^{\prime}\right)$ in column 5 of Table 2 corresponding to $\tilde{\pi}=9$ is $c+a b=w_{2}(\tilde{\pi})$, as given by Table 1. Similarly $\tilde{\pi}=8+1$ occurs twice in the fourth column of Table 2. Again, the sum of weights $\tilde{w}\left(\pi^{\prime}\right)$ in column 5 of Table 2 corresponding to $\tilde{\pi}=8+1$ is 
TABLE 2

\begin{tabular}{|c|c|c|c|c|}
\hline$\underline{\pi^{\prime}}$ & $w\left(\pi^{\prime}\right)$ & $\underline{\text { Graph }}$ & $\underline{\tilde{\pi}}$ & $\tilde{w}\left(\pi^{\prime}\right)$ \\
\hline $9_{c}$ & $c$ & ......... & $9_{c}$ & $\mathrm{c}$ \\
\hline $8_{b}+1_{a}$ & $a b$ & $\ldots \ldots \ldots$ & $9_{a b}$ & $a b$ \\
\hline $7_{a}+2_{b}$ & $a b$ & $\begin{array}{l}\ldots \ldots . . \\
. .\end{array}$ & $8_{b}+1_{a}$ & $a b$ \\
\hline $6_{c}+3_{c}$ & $c^{2}$ & $\begin{array}{l}\ldots \ldots \\
\ldots\end{array}$ & $7_{a}+2_{b}$ & $a b$ \\
\hline $6_{c}+2_{b}+1_{a}$ & $a b c$ & $\begin{array}{l}\ldots \ldots . \\
. . \\
.\end{array}$ & $8_{b c}+1_{a}$ & $a b c$ \\
\hline $5_{b}+4_{a}$ & $a b$ & $\begin{array}{l}\ldots . . \\
\ldots .\end{array}$ & $6_{c}+3_{c}$ & $c^{2}$ \\
\hline $5_{b}+3_{c}+1_{a}$ & $a b c$ & $\begin{array}{l}\ldots . . \\
\ldots\end{array}$ & $7_{a c}+2_{b}$ & $a c b$ \\
\hline $4_{a}+3_{c}+2_{b}$ & $a b c$ & $\begin{array}{l}\cdots . \\
\ldots \\
. .\end{array}$ & $6_{c}+3_{a b}$ & $c a b$ \\
\hline
\end{tabular}

$a b+a b c=(b+b c) a=w_{2}(\tilde{\pi})$, as in Table 1 . The same observations apply to the other partitions $\tilde{\pi}$ in column 4 , and so this verifies Theorem 2 for $n=9$.

It is to be noted that the weights $w\left(\pi^{\prime}\right)$ and $\tilde{w}\left(\pi^{\prime}\right)$ are not equal in general. But $w\left(\pi^{\prime}\right)$ and $\tilde{w}\left(\pi^{\prime}\right)$ have the same degree as monomials in variables $a, b$ and $c$. Thus, it is not at all obvious, and quite difficult to show, that the sum of $w\left(\pi^{\prime}\right)$ is equal to the sum of $\tilde{w}\left(\pi^{\prime}\right)$ as asserted by Theorem 8 . We have not given a combinatorial proof that the two sums are the same. Instead, we have assumed the truth of Theorem 2 (which is a partition interpretation of the key identity (1.2) proved in [3] and equivalent to (1.2)) and given a combinatorial bijection explaining Theorems 2 and 8 , both of which are equivalent to a refined form of Theorem G. Since $w\left(\pi^{\prime}\right)$ and $\tilde{w}\left(\pi^{\prime}\right)$ are not the same as functions of the variables $a, b$ and $c$, this bijection is very delicate and works only for the transformations (1.4). Under the transformations (1.3), this bijection yields a correspondence between the set of all partitions into distinct parts $\equiv 2,4$ or $5(\bmod 6)$ and the set of all partitions of the form $m_{1}+m_{2}+\ldots+m_{\nu}$ satisfying $m_{\nu} \neq 1$ or 3 , and $m_{i}-m_{i+1} \geq 6$ with strict inequality if $m_{i} \equiv 0,1$ or $3(\bmod 6)$. However, it does not yield a bijection between the sets of partitions of $n$ counted by $B(n)$ and $C(n)$ in Theorem $\mathrm{G}$. 


\section{Applications}

A simpler approach to Theorem 2 without appeal to (1.2) can be given along the following lines. I noticed recently by a further combinatorial study that Theorem 2 is equivalent to the new key identity

$$
\sum_{i, j, k \geq 0} \frac{a^{i} b^{j} c^{k} q^{T_{i+j+k}}(-c)_{i}(-c)_{j}\left(-a b c^{-1} q\right)_{k}(-c q)_{i+j}}{(q)_{i}(q)_{j}(q)_{k}(-c)_{i+j}}=(-a q)_{\infty}(-b q)_{\infty}(-c q)_{\infty}
$$

under the transformations (1.4). Subsequently, Andrews gave a $q$-series proof of (6.1) utilizing Jackson's $q$-analog of Dougall's summation. This shows that (6.1) is still quite deep, although simpler than (1.2). In a separate paper [2], the combinatorial equivalence of Theorem 2 and (6.1) as well as the $q$-series proof of (6.1) will be given.

We conclude by considering some interesting special cases of Theorem 2 .

First let $c=0$, in which case the product in (1.5) becomes

$$
\left(-a q ; q^{3}\right)_{\infty}\left(-b q^{2} ; q^{3}\right)_{\infty} .
$$

With $c=0$, the weights $w_{2}(\tilde{\pi})$ are easy to compute. For partitions $\tilde{\pi}=m_{1}+$ $m_{2}+\cdots+m_{\nu}$ with minimal difference $3, w_{2}\left(m_{i}\right)=a$ if $m_{i} \equiv 1(\bmod 3)$ and $w_{2}\left(m_{i}\right)=b$ if $m_{i} \equiv 2(\bmod 3)$ regardless of the difference $m_{i}-m_{i+1}$. On the other hand, if $m_{i} \equiv 0(\bmod 3)$, then $w_{2}\left(m_{i}\right)=0=c$ whenever $m_{i}-m_{i+1}=3$. Since $w_{2}(\tilde{\pi})$ is defined to be the product of the weights of its parts, this means that in $\tilde{\pi}$ consecutive multiples of 3 cannot occur. Thus with $c=0$, Theorem 2 yields the following refined form of Schur's celebrated 1926 partition theorem [10]:

Theorem 11. Let $S(n ; i, j)$ denote the number of partitions $\pi$ of $n$ into distinct parts such that $\nu_{1}(\pi)=i, \nu_{2}(\pi)=j$ and $\nu_{3}(\pi)=0$.

Let $S_{1}(n ; i, j)$ denote the number of partitions $\tilde{\pi}$ of $n$ with minimal difference 3 and no consecutive multiples of 3 as parts such that $\nu_{1}(\tilde{\pi})+\nu_{3}(\tilde{\pi})=i$ and $\nu_{2}(\tilde{\pi})+$ $\nu_{3}(\tilde{\pi})=j$.

Then $S(n ; i, j)=S_{1}(n ; i, j)$.

Next consider $c=-1$, in which case the product in (1.5) is

$$
\left(-a q ; q^{3}\right)_{\infty}\left(-b q^{2} ; q^{3}\right)_{\infty}\left(q^{3} ; q^{3}\right)_{\infty} .
$$

With $c=-1$, we have $a+a c=b+b c=0$. This means that in partitions $\tilde{\pi}=m_{1}+m_{2}+\cdots+m_{\nu}$ with minimal difference 3 , there are no gaps $m_{i}-m_{i+1} \geq 4$ with $m_{i} \equiv 1$ or $2(\bmod 3)$. If we now take $a b=1$, the product in $(6.3)$ becomes

$$
\left(-a q ; q^{3}\right)_{\infty}\left(-a^{-1} q^{2} ; q^{3}\right)_{\infty}\left(q^{3} ; q^{3}\right)_{\infty} .
$$

With our choices we also have $c+a b=0$. This means $\tilde{\pi}$ cannot have any gaps $m_{i}-m_{i+1} \geq 4$ with the convention that $b_{\nu+1}=-1$. Since the minimal difference between the parts of $\tilde{\pi}$ is $\geq 3$, this leaves us with only two choices for $\tilde{\pi}$.

Case 1. $m_{\nu}=1, m_{i}-m_{i+1}=3$, for $1 \leq i<\nu$.

Case 2. $m_{\nu}=2, m_{i}-m_{i+1}=3$, for $1 \leq i<\nu$.

In Case $1, \tilde{\pi}$ is the partition

$$
1+4+7+\cdots+3 \nu-2=\frac{3 \nu^{2}-\nu}{2} .
$$


The partition $\tilde{\pi}$ has weight $w_{2}(\tilde{\pi})=a^{\nu}$. In Case $2, \tilde{\pi}$ is the partition

$$
2+5+8+\cdots+3 \nu-1=\frac{3 \nu^{2}+\nu}{2}
$$

Here $w_{2}(\tilde{\pi})=b^{\nu}=a^{-\nu}$. Thus we get Jacobi's triple product identity:

$$
\left(q^{3} ; q^{3}\right)_{\infty}\left(-a q ; q^{3}\right)_{\infty}\left(-a^{-1} q^{2} ; q^{3}\right)_{\infty}=\sum_{\nu=-\infty}^{\infty} a^{\nu} q^{\left(3 \nu^{2}-\nu\right) / 2} .
$$

\section{ACKNOWLEDGEMENTS}

I would like to thank George Andrews and Basil Gordon for several helpful discussions, and the referee for suggestions to shorten the paper.

\section{REFERENCES}

1. K. Alladi, Partition identities involving gaps and weights, Trans. Amer. Math. Soc. (to appear).

2. K. Alladi, and G. E. Andrews, A new key identity for Göllnitz' (big) partition theorem in Proc. $10^{\text {th }}$ Anniv. Conf. Ramanujan Math. Soc., Contemp. Math. (to appear).

3. K. Alladi, G. E. Andrews and B. Gordon, Generalizations and refinements of a partition theorem of Göllnitz, J. Reine Angew. Math. 460 (1995), 165-188. MR 96c:11119

4. K. Alladi and B. Gordon, Generalizations of Schur's partition theorem, Manus. Math. 79 (1993), 113-126. MR 94c:11099

5. G. E. Andrews, A partition theorem of Göllnitz and related formula, J. Reine Angew. Math., 236 (1969), 18-24. MR 40:1355

6. G. E. Andrews, The theory of partitions, Encyclopedia of Mathematics and its Applications, Vol. 2, Addison-Wesley, Reading, MA (1976). MR 58:27738

7. G. E. Andrews, q-Series: Their Development and Applications in Analysis, Number Theory, Combinatorics, Physics and Computer Algebra, CBMS Regional Conf. Ser. in Math., 66 AMS, Providence, 1986. MR 88b:11063

8. D. M. Bressoud, A combinatorial proof of Schur's 1926 partition theorem, Proc. Amer. Math. Soc., 79 (1980), 333-340. MR 81f:05017

9. H. Göllnitz, Partitionen mit Differenzenbedingungen, J. Reine Angew. Math., 225 (1967), 154-190. MR 35:2848

10. I. Schur, Zur additiven Zahlentheorie, Gesammelte Abhandlungen, Vol. 2, Springer Verlag, Berlin (1973), 43-50. MR 57:2858b

11. J. J. Sylvester, A constructive theory of partitions arranged in three Acts, an Interact and an Exodion, Amer. J. Math. 5 (1882), 251-330.

Department of Mathematics, University of Florida, Gainesville, Florida 32611

E-mail address: alladi@math.ufl.edu 